\title{
Diversity of ARSACS Mutations in French-Canadians
}

\author{
I. Thiffault, M.J. Dicaire, M. Tetreault, K.N. Huang, J. Demers-Lamarche, \\ G. Bernard, A. Duquette, R. Larivière, K. Gehring, A. Montpetit, \\ P.S. McPherson, A. Richter, L. Montermini, J. Mercier, G.A. Mitchell, N. Dupré, \\ C. Prévost, J.P. Bouchard, J. Mathieu, B. Brais
}

\begin{abstract}
Background: The growing number of spastic ataxia of Charlevoix-Saguenay (SACS) gene mutations reported worldwide has broadened the clinical phenotype of autosomal recessive spastic ataxia of Charlevoix-Saguenay (ARSACS). The identification of Quebec ARSACS cases without two known SACS mutation led to the development of a multi-modal genomic strategy to uncover mutations in this large gene and explore phenotype variability. Methods: Search for SACS mutations by combining various methods on 20 cases with a classical French-Canadian ARSACS phenotype without two mutations and a group of 104 sporadic or recessive spastic ataxia cases of unknown cause. Western blot on lymphoblast protein from cases with different genotypes was probed to establish if they still expressed sacsin. Results: A total of 12 mutations, including 7 novels, were uncovered in Quebec ARSACS cases. The screening of 104 spastic ataxia cases of unknown cause for 98 SACS mutations did not uncover carriers of two mutations. Compounds heterozygotes for one missense SACS mutation were found to minimally express sacsin. Conclusions: The large number of SACS mutations present even in Quebec suggests that the size of the gene alone may explain the great genotypic diversity. This study does not support an expanding ARSACS phenotype in the French-Canadian population. Most mutations lead to loss of function, though phenotypic variability in other populations may reflect partial loss of function with preservation of some sacsin expression. Our results also highlight the challenge of SACS mutation screening and the necessity to develop new generation sequencing methods to ensure low cost complete gene sequencing.
\end{abstract}

RÉSUMÉ: Diversité des mutations responsables de l'ARSACS chez les Canadiens-Français. Contexte : Le nombre croissant de mutations du gène de l'ataxie spastique de Charlevoix-Saguenay $(S A C S)$ rapportées à travers le monde a élargi le phénotype clinique de l'ataxie spastique de CharlevoixSaguenay, une maladie autosomique récessive (ARSACS). L'identification de patients atteints d'ARSACS au Québec, qui ne sont pas porteurs de deux mutations SACS connues, a mené au développement d'une stratégie génomique multi-modale pour découvrir des mutations dans ce gène de grande taille et explorer la variabilité phénotypique. Méthode : Nous avons recherché des mutations du gène SACS en combinant différentes méthodes chez 20 patients porteurs du phénotype canadien-français classique de l'ARSACS mais non-porteurs de deux mutations connues, et un groupe de 104 cas sporadiques ou porteurs d'une ataxie spastique récessive de cause inconnue. Nous avons examiné le buvardage Western de protéines de lymphoblastes provenant de cas porteurs de différents génotypes afin de déterminer s'ils exprimaient encore la sacsine. Résultats : Douze mutations, dont 7 nouvelles mutations, ont été identifiées chez les patients atteints d'ARSACS au Québec. La recherche de 98 mutations SACS chez 104 cas d'ataxie spastique de cause inconnue n'a pas identifié de porteurs de deux mutations connues. La sacsine était peu exprimée chez des hétérozygotes composés pour une mutation SACS faux-sense. Conclusions : La taille du gène pourrait expliquer la grande diversité génotypique observée même au Québec où un grand nombre de mutations SACS sont présentes. Cette étude n'est pas en faveur d'une plus grande variabilité du phénotype de l'ARSACS dans la population canadienne-française. La plupart des mutations entraînent une perte de fonction et la variabilité phénotypique dans d'autres populations pourrait refléter une perte partielle de fonction avec préservation d'une certaine expression de la sacsine. Nos résultats soulignent également le défi du dépistage des mutations SACS et la nécessité de développer des méthodes de séquençage de nouvelle génération afin de pouvoir offrir un séquençage complet du gène à faible coût.

Can J Neurol Sci. 2013; 40: 61-66

With more than 300 affected individuals living in Quebec, autosomal recessive spastic ataxia of Charlevoix-Saguenay (ARSACS) is the most common of all inherited recessive ataxias in the province ${ }^{1}$. Though most families originate from the regions of Charlevoix and Saguenay-Lac-St-Jean (SLSJ), cases are found in other regions of Quebec. The high carrier rate for the c.8844delT spastic ataxia of Charlevoix-Saguenay (SACS) gene mutation explains the higher prevalence of ARSACS in French-Canadians ${ }^{2}$. The carrier frequency is $1 / 22$ in the SLSJ region, but is unknown in other regions of Québec ${ }^{3}$. Surprisingly,
From the Neurogenetics of Motion Laboratory (IT, MJD, MT, KNH, JDL, GB, AD, RL, BB), Neuromuscular Group, Department of Biochemistry (KG), Genome Quebec Innovation Centre (AM), Montreal; Department of Neurology and Neurosurgery (PSM,BB), Montreal Neurological Institute, McGill University; CHU Sainte-Justine (AR, LM, JM, GAM, BB), Université de Montréal, Montréal; Département des sciences neurologiques (ND, JPB), CHUQ - Hôpital de l'Enfant-Jésus, Université Laval, Québec; Clinique des Maladies Neuromusculaires (CP, JM, BB), CSSS de Jonquière, Saguenay; Fondation de l'Ataxie de Charlevoix-Saguenay-CIHR Emerging Team on rare diseases (MJD, RL, KG, PSM, JPB, JM, BB).

Received November 24, 2011. Final Revisions Submitted July 16, 2012.

Correspondence to: Bernard Brais, Neurogenetics of Motion Laboratory,

Neuromuscular Group, Montreal Neurological Institute, McGill University, 3801 University Street, Montreal, Quebec, H3A 2B4, Canada.

Email: Bernard.Brais@mcgill.ca. 
this founder mutation was never uncovered in other populations. Genetically confirmed cases of ARSACS have now been described in: Italy, Spain, Tunisia, France, Belgium, Hungary, Morocco, Turkey, Serbia, other provinces of Canada, Netherlands, United Kingdom, Algeria and Japan (Supplemental Table 1). Therefore, despite its name, ARSACS has a worldwide distribution but its prevalence is most countries is still unknown, except in the Netherlands were it may be as frequent as Friedreich Ataxia ${ }^{4}$.

Autosomal recessive spastic ataxia of Charlevoix-Saguenay (ARSACS) was first described in a cohort of French-Canadians (FC) that presented in childhood with a progressive spastic ataxia accompanied by a sensory-motor polyneuropathy and retinal changes that were referred to as "hyper myelinated fibers" ${ }^{5,6}$. The two SACS mutations c.8844delT (6594delT) and c. $7504 \mathrm{C}>\mathrm{T}(5254 \mathrm{C}>\mathrm{T})$ were found to explain 92.6 and $3.7 \%$ respectively of carrier chromosomes ${ }^{2}$. Therefore, since 2000 it was clear that other $S A C S$ mutations in this population needed to be identified. Prior to this publication, 100 SACS mutations were uncovered in 14 countries (Supplemental Table 1 - on-line only). Together, the growing number of mutation-proven cases suggests that ARSACS is one of the most common recessive spastic ataxia worldwide. The search for SACS mutations in different cohorts has also broadened the clinical spectrum of ARSACS. The retinal changes are clearly not present in all cases $^{7}$, though they may be under observed ${ }^{8}$. Cases with important cognitive impairment have also been described, but overall it is not a common feature of ARSACS ${ }^{4}$. Four cases with onset in the twenties have also been diagnosed molecularly ${ }^{9}$. The identification over years of a group of Quebec ARSACS cases with a single or no known $S A C S$ mutation led us to a systematic search for new mutations in the large SACS gene in these classical FC ARSACS cases and sporadic or recessive spastic ataxia cases of unknown cause. This study reports the development and use of various technologies to uncover a broader spectrum of SACS mutations in ARSACS in this population and the preservation of sacsin expression in some carriers of missense mutations.

\section{Materials ANd Methods}

\section{Clinical ascertainment and consent}

Through specialized ataxia clinics and treating neurologists, we identified a cohort of 20 ataxia cases that exhibited a "classic FC ARSACS phenotype", that could not be differentiated from other FC ARSACS cases clinically. This group included 15 carriers of one c.8844delT mutation and five cases with none of the two common FC mutations. Twenty-five homozygote carriers of the common c.8844delT (6594delT) were typed to establish if they carried a large genomic deletion. We also studied a group of 104 FC spastic ataxia cases with unknown genetic diagnosis to search for SACS mutations. Detailed genealogical information, medical histories, neurological examinations, electromyelogram (EMG) and nerve conduction studies were retrieved from medical charts on all participants. This project was approved by institutional Ethics Committee of the Centre de recherche du CHUM. Informed consent was obtained from all patients. All non-ataxic healthy or disease controls signed an informed consent.

\section{Screening for new SACS mutations}

Screening for the two common SACS mutations in Quebec was performed on all ARSACS cases by the Service de Génétique Médicale, CHU Sainte-Justine, Montreal, Quebec. Genomic DNA and RNA were extracted from peripheral blood lymphocytes or from saliva using the Oragene DNA or RNA extraction kit (DNA Genotek, Kanata, Canada) using standard methods. The nine SACS coding exons and flanking intronic sequences of the SACS gene [NM_014363.4] were amplified from genomic DNA using 42 primer pairs designed with UCSC ExonPrimer (http://ihg.gsf.de). RT-PCRs on RNA from white blood cells or saliva were performed using 34 primer pairs to cover the entire coding sequence. Oligonucleotide primers were synthesized by LifeTechnologies (www.lifetechnologies.com). Genomic and cDNA PCR products were forward and reverse sequenced at the McGill University and Genome Quebec Innovation Centre using an ABI 3730xl DNA Analyzer (ABI; Applied Biosystems, Foster City, CA, USA). Sequences were analyzed using SeqMan 4.03 (DNAStar, Wisconsin, USA) and Chromas 1.62 (Technelysium Pty Ltd, Australia).

\section{Custom Sequenom iPlex screening for 96 SACS variants}

To rapidly assess the frequency of known SACS mutations in sporadic or recessive spastic ataxia cases, we designed Sequenom iPlex Gold custom panels (Sequenom, San Diego, CA, USA) in collaboration with the McGill University and Genome Quebec Innovation Centre. Three panels were designed for a total of 87 SACS mutations reported at the time in the literature or uncovered by us in the FC ARSACS cohort and nine previously identified variants predicted to lead to an amino acid change or to a premature STOP codon (Supplemental Table 2 on-line only). The iPLEX® Gold assay consists in a multiplex PCR followed by a single base primer extension reaction. Following the PCR the remaining nucleotides are deactivated by Shrimp Alkaline Phosphatase (SAP) treatment. The single base primer extension step is performed, and the primer extension products are detected using MALDI TOF Mass Spectrometry. Data analysis and genotype calling was performed using Typer 4.0 Analyzer software (Sequenom, San Diego, CA, USA).

\section{Copy number variation analysis}

In order to detect deletions or duplications in SACS, DNA samples from ten ARSACS patients with a single mutation were sent to McGill University and Genome Quebec Innovation Center (Montreal, Canada) for a copy number analysis scan using Illumina Human 1M Duo that includes 108 markers in the SACS gene. Analyses of changes in gene copy number were performed using Copy Number Variation (CNV) Analysis with BeadStudio v3.1 (Illumina, San Diego, CA, USA) and PennCNV program as previously described ${ }^{10}$. To further assess small deletion or duplication in $S A C S$, we used 24 pre-designed ABI ${ }^{\circledR}$-based Copy Number Assays for human CNV screening; eleven were located in SACS coding regions, and one in each coding sequence of the surrounding genes (FGF9, SCGC, TNFRSF19, MIPEP, PCOTH, RP11-45B20.2, SPATA13 and PARP4) (Supplemental Table 3 - on-line only). Each reaction was performed in quadruplicate on 384-well PCR plates with the ABI ${ }^{\circledR}$ Copy Number Reference Assay (RNaseP). CopyCaller ${ }^{\mathrm{TM}}$ 
(Applied Biosystems, Carlsbad, CA, USA) was used for data analysis.

\section{Western blot analysis}

Proteins were extracted from immortalized lymphoblasts prepared using standard methods. Cells were trypsinized, pelleted and homogenized in 20mM HEPES buffer with protease inhibitors. Protein concentrations were determined using Bradford reagent (BioRad). Homogenates were separated on a 3-8\% Tris-Acetate gel (LifeTechnologie, www.lifetechnologies .com) and transferred onto a nitrocellulose membrane (BioRad). The membrane was then probed with a custom in-house affinity purified rabbit polyclonal anti-sacsin antibody (raised against human sacsin protein aa. 4445-4579).

\section{RESULTS}

Identification of novel SACS point mutations and genomic rearrangements in French-Canadians ARSACS cases

Since 2000, a growing number of clinically presumed FC ARSACS cases with a single mutation or no mutations were identified $^{2}$. This prompted our systematic search for novel SACS point mutations and genomic rearrangements. We sequenced based on the reference sequence [NM_014363.4] the genomic SACS coding sequence on 15 cases with a single known mutation and five with no mutations presenting classical FC ARSACS phenotype. We also sequenced the full SACS copy DNA (cDNA) on four of these cases. As summarized in the Table, both mutations were uncovered in 18/20 of the cases (90\%) (Figure 1). They consist of seven not previously described and five previously published mutations. These mutations include: five missenses, five insertions and deletions (indels), one nonsense and one large genomic deletion. In this cohort no new carriers of the previously reported c.7504C $>\mathrm{T}$ mutation was found, while all previously known carriers were excluded from this study considering that all had two known SACS mutations. The two most common other mutations appear to be c.814C $>\mathrm{T}$ (p.R272C), previously described in cases from Maritime Canada $^{11}$, and the novel c.4744G>A (p.D1582N) (Table 1). We also identified two novel mutations c.6290delG (p.C2097LfsX2113) and c.9086delA (p.N3029IfsX3051) in a Quebec resident of French-Italian origin with a classical FC ARSACS phenotype. We screened using a custom Sequenom iPlex for 87 SACS mutations in a group of 94 pan-Quebec French-Canadian healthy or diseased non-ataxic controls. We uncovered only three carriers of the common c.8844delT mutation in (1:33), and no carriers of the other mutations. This further supports that the higher prevalence of ARSACS in FC is due uniquely to the high carrier rate of the c.8844delT mutation in FC.

Evidence for a deletion of the entire SACS gene was uncovered early in this study by simple sequencing in one patient considered homozygote for c.8844delT (6594delT) and found to be homozygote for many SNP variants in the SACS

Table: Genotypes of 18 ARSACS cases carrying novel (*) or already published SACS FC mutations

\begin{tabular}{|c|c|c|}
\hline Number of cases & Mutation 1 & Mutation 2 \\
\hline 5 & c.8844delT (p.I2949FfsX2952) & c. $4744 \mathrm{G}>\mathrm{A}(\mathrm{p} . \mathrm{D} 1582 \mathrm{~N}) *$ \\
\hline 2 & c.8844delT (p.I2949FfsX2952) & c. $814 \mathrm{C}>\mathrm{T} \quad$ (p.R272C) \\
\hline 1 & c.8844delT (p.I2949FfsX2952) & c.12992G>A (p.R4331Q) \\
\hline 1 & c.8844delT (p.I2949FfsX2952) & c.237insAfsX (p.S80IfsX98)* \\
\hline 1 & c.8844delT (p.I2949FfsX2952) & c.1190insAfsX (p.S397KfsX405)* \\
\hline 1 & c.8844delT (p.I2949FfsX2952) & large del $1,41 \mathrm{Mb}$ \\
\hline 1 & c.8844delT (p.I2949FfsX2952) & c.5836T $>$ C (p.W1946R) \\
\hline 1 & c.8844delT (p.I2949FfsX2952) & c. $9508 \mathrm{C}>\mathrm{T}(\mathrm{p} . \mathrm{R} 3170 \mathrm{X}) *$ \\
\hline 1 & c.5836T $>\mathrm{C} \quad(\mathrm{p} . \mathrm{W} 1946 \mathrm{R})$ & c.237insAfsX (p.S80IfsX98)* \\
\hline 1 & c.5836T $>\mathrm{C} \quad$ (p.W1946R) & c. $4205 \mathrm{~A}>\mathrm{T} \quad(\mathrm{p} . \mathrm{D} 1402 \mathrm{~V})^{*}$ \\
\hline 1 & c.11242 del688bp (p.3748fs3756X)* & c. 11242 del688bp (p.3748fs $3756 \mathrm{X})$ \\
\hline 1 & c. $814 C>T \quad(p . R 272 C)$ & c.814C $>\mathrm{T} \quad(\mathrm{p} . \mathrm{R} 272 \mathrm{C})$ \\
\hline 1 & c.9497-9498delTT (p.F3166X)* & c.9497-9498delTT (p.F3166X)* \\
\hline
\end{tabular}






Figure 1: Schematic representation of the SACS mRNA showing the localization of 104 SACS reported mutations. Positions are based on the longest $m R N A$ transcript containing 9 coding exons numbered from 2 to 10 based on reference sequence NM_014363.4. Novel (*) French-Canadian (FC) and French-Italian (FI) mutations described in this study in Quebec ARSACS cases are identified as are previously described FC mutations. SACS sequences coding for predicted or known sacsin domains are represented as boxes. The three large deletions reported including the entire SACS gene are not illustrated. UBL: ubiquitin-like domain; SRR: sacsin repeating region; XPCB: xeroderma pigmentosum complementation groupe C binding domain; DnaJ: heat shock protein DnaJ; HEPN: higher eukaryotes and procaryotes nucleotide binding domain; bp: base pair.

gene (Table). Copy number variation (CNV) analysis using the Illumina Human 1M Duo chip established in this case the existence of a $1.41 \mathrm{Mb}$ deletion (position on chromosome 13: 23511581-24928468), which includes SACS and five other genes, which share similar general boundaries as reported in other large regional deletion ${ }^{12-14}$. This may correspond to the same historical deletion observed in a homozygote FC cases described with both ARSACS and LGMD2C due to the combined loss of SACS and $\mathrm{SGCG}^{12}$. To establish if such deletion may have led to an erroneous diagnosis of homozygote carrier for the common c.8844delT we screened 25 such ARSACS cases using 24 pre-designed ABI ${ }^{\circledR}$-based Copy Number Assays mapped to the SACS region and six surrounding genes. We were able to confirm the existence of the large deletion in our single deletion proven case (Table). No rearrangements were found in the 25 presumed homozygotes for the c.8844delT mutation, or in 15 cases with only one mutation at the onset of this study (Table). Together, these results suggest that large genomic deletions of SACS are rare in the FC population. We did observe small exonic deletions or duplications in many cases, as was described by Baets et $\mathrm{al}^{9}$, but their presence in many of 68 controls, 62 spastic ataxia and 43 patients already known for two mutations suggest that they correspond to common $\mathrm{CNV}$ in populations of European descent and should not be considered pathological mutations. Despite the different methods used, two clinically affected ARSACS cases have only one uncovered mutation. This may suggest that we have not yet found the second mutation or that in fact they are affected by other ataxias and are simply carriers of the common c.8844delT SACS mutation by chance.

\section{ARSACS phenotype in French-Canadians}

By selecting cases with a "classical FC ARSACS" phenotype with at least one unknown mutation, this study was not designed to explore phenotype/genotype correlation in FC cases with two confirmed mutations. The more overlapping phenotypes in this cohort of 18 confirmed cases probably reflects that $89 \%(16 / 18)$ carry one copy of the common FC c.8844delT mutation (n:13) or at least one nonsense mutation (n:3). We confirmed the absence of sacsin on western blot for the homozygotes for the nonsense c.8844delT mutation while there is still some degree of expression in cases with at least one missense mutation (Figure 2). This raises the possibility that some carriers of at least one missense mutation with preservation of expression of mutated sacsin may have a partial loss of function and a milder phenotype. Indeed all cases with later onset of ARSACS reported by Baets et al have at least one missense mutation ${ }^{9}$.

To explore if other FC spastic ataxia patients with nonARSACS phenotypes carried other SACS mutations we combined two methods to cheaply screen a large number of

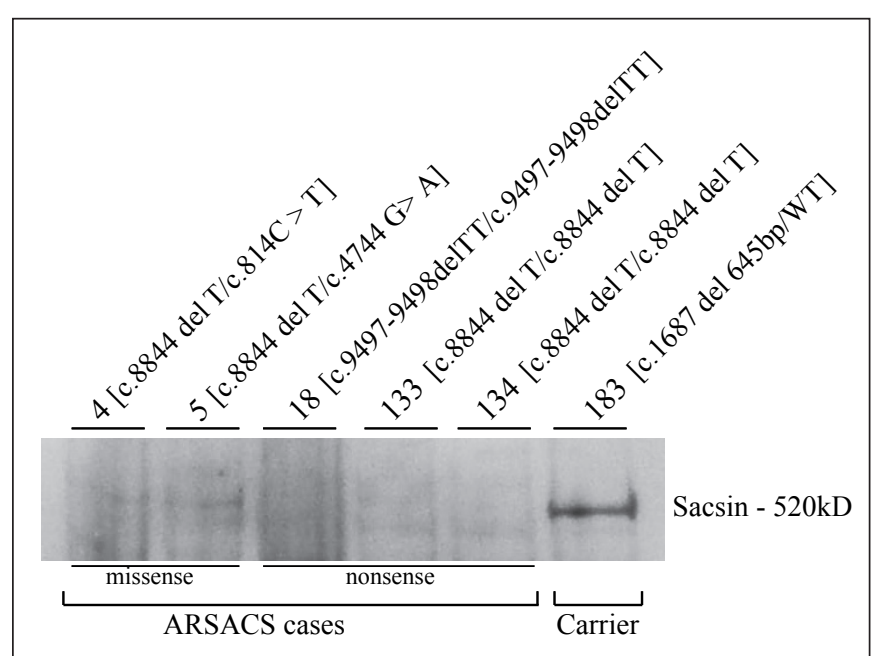

Figure 2: Western blot showing the partial preservation of sacsin in lymphosblasts from cases 4 and 5 carrying one missense mutation. 
cases for 87 SACS mutations, including the seven potential novel mutations found in our cohort, and exclude a large genomic deletion of the SACS region. First, we designed a custom Sequenom iPlex Gold technology (Sequenom, San Diego, CA, USA) based assay that screens plates of 92 samples for 87 SACS mutations. The genotype of 45 ARSACS samples was successfully confirmed using this assay. Using this technology we also screened for SACS mutations 104 samples from a heterogeneous group of FC spastic ataxic patients without a molecular diagnosis. One carried the common c.8844delT, while two were heterozygotes for the c.4744G $>$ A. Furthermore, on 62 samples, none were found to carry a genomic regional SACS rearrangement using a custom $\mathrm{ABI} 囚$-based Copy Number Assays for human $\mathrm{CNV}$ assay, further supporting that this deletion is rare in $\mathrm{FC}$.

\section{Discussion}

This study establishes that even within a founder population like the French-Canadians there is a great diversity of SACS mutations that cause a "classical FC ARSACS" phenotype. Though clearly the higher prevalence of ARSACS in this population is due to the high carrier rate of the common c.8844delT (6594delT) mutation, five other mutations are also recurrent: c.7504C $>$ T, c. $4744 \mathrm{G}>\mathrm{A}$, c $.814 \mathrm{C}>\mathrm{T}, \mathrm{c} .237$ ins Afs $\mathrm{X}$ and c. $5836 \mathrm{~T}>\mathrm{C}$. In the case of the $\mathrm{c} .814 \mathrm{C}>\mathrm{T}$ mutation, this may well be due to an Acadian introduction considering that the same mutation was found in Maritime Canada ${ }^{11}$. As observed in other populations, many mutations appear to be private familial mutations. This mutation diversity and positional scattering along the gene suggests that the large size of SACS by itself is responsible for the large number of mutations (Figure 1). Large genomic deletions that include $S A C S$ and other neighbouring genes have now been reported by many groups ${ }^{12-14}$. It is still unknown if these are all independent deletion events or a few rare historical deletions shared by all these cases. More detailed comparisons of the break points will be needed to settle this issue. Our study demonstrates that these large deletions are not common in the French-Canadian population, by this supporting that PCR based detection of the common SACS mutations are unlikely to have missed heterozygotes for a point mutations and a large deletion. Significantly, the uncovering that an homozygote carrier of two large deletions suffers from a combined ARSACS and LGMD2C established that a complete knock out of sacsin is not lethal ${ }^{12}$. The growing number of missense SACS mutations suggests that predicted partial loss of function may lead to different ARSACS phenotypes.

Now that clinicians are aware that ARSACS may be a common form of spastic ataxia worldwide, the challenge is to ensure that its molecular diagnosis can be readily performed at low cost. The multimodal approach used in this study provides a way to more rapidly screen larger numbers of samples for a wider range of mutations than the previously proposed Confirmation Sensitive Capillary Electrophoresis-Based Mutation Scanning System (CSCE) ${ }^{15}$. The screening for common mutations in certain populations by inexpensive PCRbased methods should still be the first step in establishing a diagnosis. However, with the declining cost of new generation sequencing and the increasing number of SACS mutations being described, we would favour the full sequencing of the gene combined to $\mathrm{CNV}$ analysis if one parent is not found to carry the child's presumed homozygote mutation as the way to establish if patients are affected by ARSACS.

The growing number of reported ataxic patients who carry two SACS mutations that do not have a "classical FC ARSACS" phenotype in other countries has expanded the sacsinopathy phenotypes ${ }^{9}$. However, our screening of 104 FC spastic ataxia cases of unknown cause did not uncover cases with two SACS mutations. Therefore, in this population ARSACS presents a relatively homogenous phenotype likely because of the predominant common c.8844delT (6594delT) mutation. The high prevalence of ARSACS and Friedreich ataxia in Quebec justifies that mutations in both SACS and FXN genes should be done first in all FC childhood-onset cases of spastic ataxia with a polyneuropathy. Other genes that can be screened in FC childhood-onset ataxia cases considering their relatively higher frequencies in this population are: the SETX gene in cases with a polyneuropathy without significant spasticity responsible for Ataxia Oculomotor Apraxia $2(\mathrm{AOA} 2)^{16}$, and the MARS2 gene responsible for the very variable Autosomal recessive Spastic Ataxia with frequent Leukoencephalopathy (ARSAL) in which a polyneuropathy is not a feature in the early stages of the disease $^{17,18}$. This study confirms that in ARSACS, as for all other ataxias with higher regional prevalence in Quebec described to date such as AOA2 ${ }^{16}$, ARSAL ${ }^{17,18}$ and Autosomal Recessive Cerebellar Ataxia (ARCA1) 19,20 , there is a wide spectrum of mutations. However, as for the other ataxias with Quebec founder effects, despite the large number of living cases, these cohorts have a limited power for genotype/phenotype correlation because of the high prevalence of a common founder mutation. Our observation that some sacsin is present in carriers of at least one missense mutation raise the possibility that partial preserved function can influence severity. In fact, all cases with later onset of ARSACS reported by Baets et al have at least one missense mutation ${ }^{9}$. Clinical and mutation data sets from combined international series are required to complete a definitive genotype/phenotype correlation. Though uncovering how SACS mutations lead to ARSACS was hampered by the large size of the sacsin protein $(520 \mathrm{KDa})$, recent studies have started to unravel sacsin's potential dimeric structure ${ }^{21}$ and its chaperone function $^{22-24}$. Furthermore, the Fondation de l'Ataxie de Charlevoix-Saguenay-CIHR ARSACS Emerging Team recently established that sacsin localizes to the surface of mitochondria and that knock down or knock out of sacsin in cellular and mice models lead to a disruption of mitochondrial transport into dendrites with ensuing abnormal dendritic morphology in neurons, in particular in Purkinje cells of the cerebellum, that precedes their death ${ }^{25}$. Therefore, mitochondrial dysfunction/ mislocalization are the likely cellular bases for ARSACS, supporting that, as in Friedreich ataxia and a growing of other ataxias and neurodegenerative disorders, mitochondrial dysfunction plays a central role in their pathophysiology ${ }^{26}$.

\section{ACKNOWLEDGEMENTS}

The authors thank all cases and their families for their essential participation and the staff of the different clinics for their support. We would also like to thank colleagues who provided samples. We acknowledge the technical support of the McGill University and Genome Quebec Innovation Centre for 
their support in the sequencing. This project was generously supported by the Fondation de l'Ataxie Charlevoix-Saguenay (www.arsacs.com) and the Neuromuscular Research Partnership Program of the Canadian Institutes of Health Research (CIHR), Muscular Dystrophy Canada (MDC) and the ALS Society of Canada. Many of the authors are new members of the Fondation de l'Ataxie de Charlevoix-Saguenay-CIHR ARSACS Emerging Team. G. Bernard received a scholarships from the RMGA (Réseau de médecine génique appliquée du FRSQ) and the FRSQ (Fonds de Recherche en Santé du Québec). I. Thiffault received scholarships from the Fondation de l'Ataxie Charlevoix-Saguenay, the CIHR and the Montreal Neurological Institute ETP fellowship from the National Bank Financial Group. M. Tetreault received a scholarship from the CIHR.

\section{REFERENCES}

1. Dupré N, Bouchard JP, Brais B, Rouleau GA. Hereditary ataxia, spastic paraparesis and neuropathy in the French-Canadian population. Can J Neurol Sci. 2006;33:149-57.

2. Engert JC, Berube P, Mercier J, et al. ARSACS, a spastic ataxia common in northeastern Quebec, is caused by mutations in a new gene encoding an 11.5-kb ORF. Nat Genet. 2000;24:120-5.

3. De Braekeleer M, Giasson F, Mathieu J, Roy M, Bouchard JP, Morgan K. Genetic epidemiology of autosomal recessive spastic ataxia of Charlevoix-Saguenay in northeastern Quebec. Genet Epidemiol. 1993;10:17-25.

4. Vermeer S, Meijer RP, Pijl BJ, et al. ARSACS in the Dutch population: a frequent cause of early-onset cerebellar ataxia. Neurogenetics. 2008;9:207-14.

5. Bouchard JP, Barbeau A, Bouchard R, Bouchard RW. Autosomal recessive spastic ataxia of Charlevoix-Saguenay. Can J Neurol Sci. 1978;5:61-9.

6. Bouchard JP, Barbeau A, Bouchard R, Bouchard RW. Electromyography and nerve conduction studies in Friedreich's ataxia and autosomal recessive spastic ataxia of CharlevoixSaguenay (ARSACS). Can J Neurol Sci. 1979;6:185-9.

7. Okawa S, Sugawara M, Watanabe S, Imota T, Toyoshima I. A novel sacsin mutation in a Japanese woman showing clinical uniformity of autosomal recessive spastic ataxia of CharlevoixSaguenay. J Neurol Neurosurg Psychiatry. 2006;77:280-2.

8. Desserre J, Devos D, Sautiere BG, et al. Thickening of peripapillar retinal fibers for the diagnosis of autosomal recessive spastic ataxia of Charlevoix-Saguenay. Cerebellum. 2011 Dec;10(4): 758-62.

9. Baets J, Deconinck T, Smets K, et al. Mutations in SACS cause atypical and late-onset forms of ARSACS. Neurology. 2010;75: 1181-8.

10. Wang K, Li M, Hadley D, et al. PennCNV: an integrated hidden Markov model designed for high-resolution copy number variation detection in whole-genome SNP genotyping data. Genome Res. 2007;17:1665-74.
11. Guernsey DL, Dube MP, Jiang H, et al. Novel mutations in the sacsin gene in ataxia patients from Maritime Canada. J Neurol Sci. 2010;288:79-87.

12. McMillan HJ, Carter MT, Jacob PJ, Laffan EE, O'Connor MD, Boycott KM. Homozygous contiguous gene deletion of $13 q 12$ causing LGMD2C and ARSACS in the same patient. Muscle Nerve. 2009;39:396-9.

13. Breckpot J, Takiyama Y, Thienpont B, et al. A novel genomic disorder: a deletion of the SACS gene leading to spastic ataxia of Charlevoix-Saguenay. Eur J Hum Genet. 2008;16:1050-4.

14. Terracciano A, Casali C, Grieco GS, et al. An inherited large-scale rearrangement in SACS associated with spastic ataxia and hearing loss. Neurogenetics. 2009;10:151-5.

15. Vermeer S, Meijer RPP, Hofste TGJ, et al. Design and validation of a conformation sensitive capillary electrophoresis -based mutation scanning system and automated data analysis of the more than $15 \mathrm{kbp}$-spanning coding sequence of the SACS Gene. J Mol Diagn. 2009;11:514-23.

16. Duquette A, Roddier K, McNabb-Baltar J, et al. Mutations in senataxin responsible for Quebec cluster of ataxia with neuropathy. Ann Neurol. 2005 Mar;57(3): 408-14.

17. Bayat $\mathrm{V}$, Thiffault I, Jaiswal $\mathrm{M}$, et al. Mutations in the mitochondrial methionyl-tRNA synthetase cause a neurodegenerative phenotype in flies and a recessive ataxia (ARSAL) in humans. PLoS Biol. 2012;10:e1001288.

18. Thiffault I, Rioux MF, Tetreault M, et al. A new autosomal recessive spastic ataxia associated with frequent white matter changes maps to 2q33-34. Brain. 2006;129:2332-40.

19. Dupré N, Gros-Louis F, Chrestian N, et al. Clinical and genetic study of autosomal recessive cerebellar ataxia type 1 . Ann Neurol. 2007;62:93-8.

20. Gros-Louis F, Dupré N, Dion P, et al. Mutations in SYNE1 lead to a newly discovered form of autosomal recessive cerebellar ataxia. Nat Genet. 2007 Jan;39(1):80-5.

21. Kozlov G, Denisov AY, Girard M, et al. Structural basis of defects in the sacsin HEPN domain responsible for autosomal recessive spastic ataxia of Charlevoix-Saguenay (ARSACS). J Biol Chem. 2011 Jun 10;286(23):20407-12.

22. Parfitt DA, Michael GJ, Vermeulen EG, et al. The ataxia protein sacsin is a functional co-chaperone that protects against polyglutamine-expanded ataxin-1. Hum Mol Genet. 2009;18: 1556-65.

23. Anderson JF, Siller E, Barral JM. The sacsin repeating region (SRR): a novel Hsp90-related supra-domain associated with neurodegeneration. J Mol Biol. 2010;400:665-74.

24. Anderson JF, Siller E, Barral JM. The neurodegenerative disease related protein sacsin is a molecular chaperone. J Mol Biol. 2011 Aug 26;411(4):870-80.

25. Girard M, Larivière R, Parfitt DA, et al. Mitochondrial dysfunction and Purkinje cell loss in autosomal recessive spastic ataxia of Charlevoix-Saguenay (ARSACS). Proc Natl Acad Sci U S A. 2012 Jan 31;109(5):1661-6. Epub 2012 Jan 17.

26. Schon EA, Przedborski S. Mitochondria: the next (neurode) generation. Neuron. 2011;70:1033-53. 